SNPs genotyping TaqMAN technology and we respected the Hardy-Weinberg equilibrium.

Results First, we analyzed the allele frequency of NOS2 and VEGF genes SNPs and we obtained for:

NOS2 gene that rs2779248 T allele is associated to SLE's development (OR 1.92) as well as rs2779251 T (OR 2.01) and rs8078340 A alleles (OR 5.00) unlike rs2779248 C, rs2779251 $\mathrm{G}$ and rs8078340 $\mathrm{G}$ alleles that are protective against SLE development (OR 0.52, 0.50 and 0.20).

VEGF gene that rs2010963 C allele is associated SLE's development (OR 1.86) unlike rs2010963 G allele that is protective (OR 0.54).

Thereafter, we analyzed the genotype frequency and we got for:

NOS2 gene that rs2779248 CT genotype is associated to SLE's development (OR 2.01) as well as rs2779251 GG (OR 1.62) and rs8078340 AA genotypes (OR 3.41) inversely to rs2779248 CC, rs2779251 TT and rs8078340 GG genotypes that are protective (OR $0.35,0.24$ and 0.20 ).

VEGF gene that rs1570360 GG genotype is associated to SLE's development (OR 1.73) as well as rs2010963 CC genotype (OR 2.91) unlike rs1570360 AG and rs2010963 GG genotypes that are protective (OR 0.51 and 0.58 ).

Furthermore, we observed that the VEGF rs1570360 G allele was assiociated to lupus nephritis development (OR 3.51) as well as GG genotype (OR 3.82). Regarding the haplotype analysis, it showed for the NOS2 gene that AGG haplotype is associated to SLE's development (OR 2.12) and that CGG is protective (OR 0.50). Finally, as a whole, our results are consistent with the literature data.

Conclusions At the end of our study, we have demonstrated the role the NOS2 and VEGF genes SNPs in the genetic susceptibility to develop SLE in Algerian patients.

Funding Source(s): Pasteur Institute of Algeria

\section{METFORMIN ENHANCES ANTI-INFLAMMATORY EFFECTS OF ADIPOSE-DERIVED MESENCHYMAL STEM CELLS: THERAPEUTIC POTENTIAL OF METFORMIN-TREATED AD- MSCS IN ANIMAL MODEL OF LUPUS}

Se Gwang Jang*, Jaeseon Lee, Seung-Min Hong, Seung-Ki Kwok, Sung-Hwan Park. The Catholic University

\subsection{6/lupus-2019-Ism.120}

Background Metformin is originally introduced as a biguanide antidiabetic medication, has an anti-inflammatory effect via activating AMP-activated protein kinase (AMPK). Human adipose-derived stem cells (Ad-MSCs) are stromal cells derived from adipose tissue and known to have immunoregulatory activity. The study was undertaken to examine whether metformin-treated Ad-MSCs show more potent therapeutic effect in animal model of lupus and to clarify the underlying mechanism of potent immunoregulatory impact of metformin-treated Ad-MSCs.

Methods To examine the effects of metformin, Ad-MSCs were incubated for 72 hour in the presence of metformin. Cellular phenotype of Ad-MSCs was analyzed by flow cytometry. Indoleamine 2,3-dioxygenase, IL-10 and TGF-1 expression was analyzed by real-time PCR and ELISA. AMPKmTOR pathway was analyzed by Western blotting in AdMSCs with or without metformin. MRL/lpr mice weekly injected $1 \times 10^{\wedge}$ metformin-treated Ad-MSCs in $0.1 \mathrm{ml}$ PBS to lateral tail vein for 7 weeks. All mice were sacrificed at the age of 16 weeks. Urinary albumin-to-creatinine ratios were then calculated. The amount of anti-dsDNA IgG antibody in mice sera was measured by ELISA. PAS stained kidney sections were used for assessment of histology. Deposition of IgG and C3 was detected by confocal microscope. Population of cellular subset in spleen, kidney and blood was analyzed by Flow cytometry.

Results In vitro, metformin-treated Ad-MSCs increased mRNA level of IDO, IL-10 and TGF-1 compared with untreated Ad-MSCs. Also, the concentrations of IDO, IL-10 and TGF- increased in culture supernatants. Metformin upregulated expression of p-AMPK and inhibited the expression of p-STAT3, p-mTOR, and p-Raptor in AdMSCs. Intravenously injected metformin-treated Ad-MSCs significantly reduced the splenomegaly and lymphadenopathy compared with untreated Ad-MSCs in MRL/lpr mice. In addition, Metformin-treated Ad-MSCs decreased anti-dsDNA antibodies in serum and proteinuria compared with untreated Ad-MSCs. Metformin-treated Ad-MSCs alleviated lupus nephritis, as judged by changes in the histopathology scores and immune complex deposition. Metformin-treated Ad-MSCs reduced CD90.2+T cells, CD90.2+CD4 CD8(double negative) $\mathrm{T}$ cells, whereas CD4 +CD25+Foxp3 + Treg cells were increased in splenocytes, kidney tissue and blood cells of MRL/lpr mice.

Conclusions Metformin can optimize the immunomodulatory potential of Ad-MSCs, suggesting a promising strategy of MSC use in lupus treatment.

Funding Source(s): None

\section{COMPARISON OF CLINICAL AND LABORATORY PROFILES IN 3575 SYSTEMIC LUPUS ERYTHEMATOSUS PATIENTS WITH AND WITHOUT SJÖGRENS SYNDROME: DATA FROM THE SPANISH SOCIETY FOR RHEUMATOLOGY LUPUS REGISTRY}

${ }^{1} J u a n$ Ovalles-Bonilla*, ${ }^{2}$ Jose Maria Pego Reigosa, ${ }^{3}$ Iñigo Rua Figueroa, ${ }^{4}$ Maria Galindo Izquierdo, 5Jaime Calvo-Alén, 1 Juan Carlos Nieto, ${ }^{1} J u l i a$ Martínez-Barrio, ${ }^{1}$ Roberto González, ${ }^{1}$ Belen Serrano, ${ }^{1}$ lustina Janta, ${ }^{1}$ Carlos M Gonzalez, ${ }^{1}$ Indalecio Monteagudo, ${ }^{6} J a v i e r \quad N a r v a ́ e z-G a r c i ́ a,{ }^{7} J o s e ́$ Luis Andréu-Sánchez, ${ }^{8}$ Mireia Moreno-Martínez-Losa, ${ }^{9}$ Ana Sánchez-Atrio, ${ }^{10}$ Loreto Horcada, ${ }^{11}$ Tatiana CoboIbáñez, ${ }^{12}$ Carlos Marras-Fernández-Cid, ${ }^{13}$ Eva Salgado-Pérez, ${ }^{14}$ Juan J Alegre-Sancho, ${ }^{1}$ Francisco Javier López-Longo. ${ }^{1}$ Hospital General Universitario Gregorio Marañón; ${ }^{2}$ Complexo hospitalario Universitario Vigo; ${ }^{3}$ Hospital de Gran Canaria dr. Negrín; ${ }^{4}$ Hospital Universitario 12 De Octubre; ${ }^{5}$ Hospital Universitario Araba; ${ }^{6}$ Bellvitge University Hospital, Barcelona; ${ }^{7}$ Hospital Universitario Puerta de Hierro; ${ }^{8}$ Hospital Parc Tauli; ${ }^{9}$ Hospital Príncipe De Asturias; ${ }^{10}$ Complejo Hospitalario de Navarra; ${ }^{11}$ Hospital Infanta Sofía; ${ }^{12}$ Hospital Virgen De La Arrixaca; ${ }^{13}$ Complejo Hospitalario de Ourense; ${ }^{14}$ Hospital dr. Peset

\subsection{6/lupus-2019-Ism.121}

Background The clinical coexistence of Systemic Lupus Erythematosus (SLE) and Sjögrens Syndrome (SS) was recognized in 1959. The prevalence of SS among patients with SLE varies considerably among the published studies (10\%-30\%). There is still controversy as to whether or not SLE patients with overlapping SS have a distinct and significantly milder lupus. To address the clinical and serologic features of SLE and differences from SLE that occurs in overlap with SS.

Methods This is a multicenter, descriptive, cross-sectional study of 3575 patients from the Spanish Society for Rheumatology Lupus Registry (RELESSER). Unselected SLE patients from 45 Rheumatology Departments across Spain were evaluated for 
Abstract 121 Table 1

\begin{tabular}{|l|c|c|c|}
\hline \multicolumn{1}{|c|}{ FEATURES } & $\begin{array}{c}\text { SLEwSS } \\
\mathbf{N}=516\end{array}$ & $\begin{array}{c}\text { SLEwoSS } \\
\mathbf{N}=3059\end{array}$ & $\mathbf{p}$ \\
\hline Age, years \pm SD & $54.2 \pm 14.9$ & $45.6 \pm 14.5$ & $<0.001$ \\
\hline Age at SLE onset, years \pm SD & $38.6 \pm 15$ & $32.1 \pm 14$ & $<0.001$ \\
\hline Female & 97.5 & 89.1 & $<0.001$ \\
\hline Lymphadenopathy & 11.8 & 10.0 & 0.233 \\
\hline Photosensitivity & 65.0 & 57.2 & 0.002 \\
\hline Oral ulcers & 55.8 & 42.7 & $<0.001$ \\
\hline Alopecia & 36.3 & 32.0 & 0.069 \\
\hline Raynaud's Phenomenon & 39.9 & 32.7 & 0.001 \\
\hline Systemic vasculitis & 9.6 & 8.4 & 0.352 \\
\hline Arthritis & 75.0 & 76.5 & 0.467 \\
\hline Myositis & 4.0 & 3.4 & 0.515 \\
\hline Fibromyalgia & 13.9 & 5.1 & $<0.001$ \\
\hline Pleurisy & 22.7 & 22.4 & 0.893 \\
\hline Pulmonary fibrosis & 2.3 & 1.9 & 0.563 \\
\hline Renal manifestations & 18.3 & 32.7 & $<0.001$ \\
\hline Seizures & 6.3 & 6.6 & 0.805 \\
\hline Peripheral neuropathy & 5.4 & 2.9 & 0.004 \\
\hline Hemolytic anemia & 7.1 & 8.6 & 0.272 \\
\hline Leucopenia & 55.6 & 53.5 & 0.428 \\
\hline Thrombocytopenia & 15.5 & 20.8 & 0.007 \\
\hline Anti-Ro/SSA & 69.2 & 34.4 & $<0.001$ \\
\hline Anti-La/SSB & 48.1 & 14.4 & $<0.001$ \\
\hline Anti-Sm & 19.8 & 21.2 & 0.495 \\
\hline Anti-RNP & 23.2 & 25.2 & 0.317 \\
\hline Anti-DNA & 55.9 & 65.1 & $<0.001$ \\
\hline Anti- $\beta 2$-GPI IgM & 8.6 & 14.5 & 0.006 \\
\hline Antiphospholipid syndrome & 13.0 & 14.0 & 0.520 \\
\hline Hypocomplementemia & 62.3 & 69.0 & 0.012 \\
\hline SLICC-ACR DI & $1.19 \pm 1.8$ & $1.03 \pm 1.7$ & 0.062 \\
\hline Neoplasia & 7.8 & 5.5 & 0.042 \\
\hline Exitus & 7.2 & 6.0 & 0.324 \\
\hline Age at death, years \pm SD & $67.3 \pm 14.4$ & $54.2 \pm 18.0$ & $<0.001$ \\
\hline SLEwSS: Systemic lupus erythematosus with Sjögren's syndrome. SLEwoSS: Systemic \\
\hline lupus erythematosus without Sjogren's syndrome. SucC-ACR DI: Systemic Lupus \\
\hline International Collaborating Clinics/American College of Rheumatology Damage Index. \\
\hline \multicolumn{1}{|c|}{ Except where indicated otherwise, values are the percentage. } \\
\hline
\end{tabular}

the presence of overlapping SS using the American-European consensus criteria. Cumulative clinical data were collected at the moment of the last assessment. Clinical and laboratory parameters in SLE patients with SS (SLEwSS) were compared with those in SLE patients without SS (SLEwoSS).

Results SS was identified in 516 SLE patients (14.4\%). Compared with the SLEwoSS group, patients with SLEwSS were significantly older, had a higher frequency of mucocutaneous manifestations, Raynauds phenomenon, peripheral neuropathy, anti-Ro/SSA, anti-La/SSB, neoplasia, and older age at death, but had a significantly lower frequency of renal involvement, thrombocytopenia, anti-dsDNA, anti-2-GPI IgM and complement consumption. Both groups displayed a clinically similar presentation of lymphadenopathy, systemic vasculitis, serositis, damage accrual, mortality, musculoskeletal and CNS manifestations.

Conclusions SLEwSS appears to constitute a subgroup of SLE patients with distinct clinical and serologic features, in whom SS is expressed as an overlapping entity. A particular cluster of clinical variables, namely, mucocutaneous manifestations, 
Raynauds phenomenon, peripheral neuropathy, renal involvement and thrombocytopenia, was found to be important overall for discriminating SLE patients with or without SS. SLEwSS patients constitute a subgroup of patients with SLE characterized by milder lupus: older age at death, similar rates of mortality and SLICC-ACR damage index, less renal and immunological manifestations.

Funding Source(s): None

\section{PERSISTENCE OF ANTI-SMITH ANTIBODY IS ASSOCIATED WITH DISEASE ACTIVITY IN PATIENTS WITH NEW-ONSET SYSTEMIC LUPUS ERYTHEMATOSUS}

Sung Soo Ahn*, Seung Min Jung, Yong-Beom Park. Yonsei University College of Medicine

\subsection{6/lupus-2019-Ism.122}

Background Anti-Smith ( $\mathrm{Sm}$ ) antibody is highly specific antibody for systemic lupus erythematosus (SLE). We evaluated the association between anti-Sm antibody and disease activity in patients with new-onset SLE.

Methods Patients who were repeatedly tested for anti-Sm antibody at SLE diagnosis and within 12 months were included in this study. The clinical and laboratory profiles, and systemic lupus erythematosus disease activity index (SLEDAI) were collected at the time of anti-Sm antibody test. SLEDAI and laboratory variables associated with disease activity were compared between patients with and without anti-Sm antibody.

Results Of 92 patients who were tested for anti-Sm antibody at SLE diagnosis, 67 patients were followed up for presence of anti-Sm antibody at 6 months, and 67 patients were followed up at 12 months. Although the baseline SLEDAI was comparable in SLE patients with or without anti-Sm antibody, immunologic and hematologic disorder was more common in anti-Sm positive patients. Patients who showed positive result of anti-Sm antibody at 6 and 12 months had higher SLEDAI compared to patients with negative result $(p=0.004$ and 0.002 at 6 and 12 months, respectively). The changes in anti-Sm antibody for 12 months was significantly correlated with the changes of SLEDAI $(p=0.029)$.

Conclusions Persistence of anti-Sm antibody for 12 months was associated with higher disease activity at the corresponding time. Follow-up of anti-Sm antibody can be useful to evaluate the remained disease activity in patients with new-onset SLE. Funding Source(s): None

\section{CLINICAL CHARACTERISTICS OF PATIENTS WITH SYSTEMIC LUPUS ERYTHEMATOSUS SHOWING THE FALSE POSITIVE RESULT OF SYPHILIS TEST}

Sung Soo Ahn*, Seung Min Jung, Yong-Beom Park. Yonsei University College of Medicine

10.1136/lupus-2019-|sm. 123

Background False positive result of syphilis test is a characteristic finding in patients with systemic lupus erythematosus (SLE), especially combined with antiphospholipid syndrome (APS). We evaluated the clinical characteristics in SLE patients who showed the false positive result of syphilis test.

Methods Patients who were tested for syphilis screening test at SLE diagnosis in Severance Hospital between January 2006 and December 2016 were included in this study. The baseline characteristics and clinical outcomes were compared between patients with false positive result of syphilis test and negative result of syphilis.

Results Of 145 patients included in this study, 20 (13.8\%) patients showed the false positive result of syphilis test. At SLE diagnosis, patients with negative syphilis result had higher SLE disease activity index $(5.0$ vs $8.0, \mathrm{p}<0.001)$, and were more commonly complicated with nephritis $(15.0 \%$ vs $41.6 \%$, $\mathrm{p}=0.026)$. Low disease activity, high protein level, and presence of APS antibodies were independently associated with the false positive result of syphilis test $(p=0.030,0.014$ and 0.002 , respectively). Although the thrombotic risk was significantly higher in patients with false positive syphilis result $(p=0.041)$, the overall mortality showed no difference between patients with false positive result and negative result of syphilis test.

Conclusions Clinical characteristics of SLE patients with false positive result of syphilis test showed lower disease activity at SLE diagnosis, but comparable overall survival and higher thrombotic risk.

Funding Source(s): None

\section{ANTIRIBOSOMAL P AUTOANTIBODIES TARGET THE NEURONAL-SURFACE-P-ANTIGEN (NSPA) IN KIDNEY AND LIVER CELLS}

${ }^{1}$ Marcela Bravo-Zehnder*, ${ }^{2}$ Carmen Sofia Espinoza, ${ }^{3}$ Patricia Gajardo, ${ }^{3}$ Tomás Toledo, ${ }^{3}$ Javiera Alvarez, ${ }^{3}$ Mariana Labarca, ${ }^{1}$ Loreto Massardo, ${ }^{4}$ Alfonso González. ${ }^{1}$ Centro de Biología Celular y Biomedicina (CEBICEM). Faculty of Science and Medicine, Universidad San Sebastián. Santiago, Chile; ${ }^{2}$ Pontificia Universidad Católica de Chile; ${ }^{3}$ Universidad San Sebastián; ${ }^{4}$ Centro de Biología Celular y Biomedicina. Faculty of Science and Medicine, Universidad San Sebastián. Santiago, Chile. Centro de Envejecimiento y Regeneración (CARE), Facultad de Ciencias, Pontificia Universidad Católica de Chile. Santiago, Chile

\subsection{6/lupus-2019-Ism.124}

Background Anti-ribosomal P (anti-P) antibodies have long been associated with lupus psychosis and recently with cognitive deficit in patients with systemic lupus erythematosus (SLE). We previously described a neuronal-surface-P-antigen (NSPA) that mediates anti-P pathogenic effects leading to memory deficit in mice. Clinical controversial associations of anti-P are lupus hepatitis and lupus nephritis (LN), in which the ribosomal $\mathrm{P} 0$ protein has been postulated as a cell surface anti-P target. As there is no mechanism explaining for the presence of $\mathrm{P} 0$ at the cell surface, we assess whether NSPA might be the anti-P cell surface target in liver and kidney cells.

Methods NSPA expression: i) RT-PCR and immunoblot in liver HepG2 and kidney HK-2 cell lines and liver and kidney tissues from C57wt and transgenic C57NSPA/KO (LacZ gene instead NSPA gene) mice; ii) ß-galactosidase histochemistry as a marker of NSPA expression in liver and kidney slices from a ZZEF-1/lac Z knock in mice; iii) Cell surface biotinylation and surface immuno-capture in combination with metabolic labeling. Functional assays: iv) Internalization assays with I125anti-P; v) Indirect immunofluorescence of activated caspase-3 and Hoechst staining of apoptotic nuclei on HK-2 treated with rabbit anti-P for 24 hour.

Results NSPA is expressed in hepatocytes, in proximal epithelium tubule renal cells and in some collecting tubules. HK-2 and HepG2 express NSPA, but not P0 at the cell surface. Anti-P bind NSPA and become internalized in a time and concentration dependent manner. Anti-P induced HK-2 apoptosis in vitro assessed by caspase- 3 activation. 